\title{
Impact of aortic stenting on peripheral vascular function and daytime systolic blood pressure in adult coarctation
}

\author{
S S M Chen, ${ }^{1}$ A E Donald, ${ }^{2}$ C Storry, ${ }^{3}$ J P Halcox, ${ }^{2}$ P Bonhoeffer, ${ }^{4}$ J E Deanfield ${ }^{2}$
}

See Editorial, p 828

${ }^{1}$ Grown-up Congenital Heart Unit, The Heart Hospital, University College London, London, UK: ${ }^{2}$ Vascular

Physiology Unit, Institute of Child Health, University College London, London, UK; ${ }^{3} \mathrm{MRC}$ Childhood Nutrition Research Centre and Vascular Physiology Unit, Institute of Child Health, University College London, London, UK; ${ }^{4}$ Great Ormond Street Hospital for Children NHS Trust and Institute of Child Health, University College London, London, UK

Correspondence to: Professor J E Deanfield, Vascular Physiology Unit, 30 Guildford Street, London WC1N 1EH, UK; j.deanfield@ich.ucl.ac.uk

Accepted 3 July 2007 Published Online First 8 August 2007

\section{ABSTRACT}

Objectives: To determine the relation of ambulatory systolic blood pressure to aortic obstruction and more extensive vascular dysfunction, assessed by central aortic, peripheral conduit arterial and resistance vessel function.

Methods: 12 adults (5 native, 7 recoarctation) were studied before, and 2 weeks and 6 months after aortic stenting. Systolic blood pressure was measured during normal daily living by 24-hour ambulatory monitoring. Central aortic function was assessed by pulse wave analysis (augmentation index). Brachial artery flowmediated dilatation and dilatation in response to $25 \mu \mathrm{g}$ of sublingual glyceryl trinitrate was assessed by ultrasound to measure peripheral conduit arterial and resistance vessel function. Baseline vascular measures were compared with those of 12 matched controls.

Results: Patients had a higher augmentation index, impaired endothelium-dependent and -independent dilatation, and forearm vascular resistance $(p<0.02)$. After successful gradient relief by stenting, daytime ambulatory systolic blood pressure $(151(134,166) \mathrm{mm} \mathrm{Hg}$ vs 138 $(130,150) \mathrm{mm} \mathrm{Hg}, \mathrm{p}=0.01)$ and the augmentation index $(26(15,34)$ vs $23(13,30), p=0.03)$ fell progressively over 6 months, but did not completely normalise. Endothelium-dependent and -independent dilatation, and forearm vascular resistance remained unchanged and impaired.

Conclusion: Relief of aortic obstruction is associated with improvement in central aortic function and results in reduction of daytime ambulatory systolic blood pressure. Peripheral vascular dysfunction, however, remains unchanged and may contribute to residual hypertension.

Arterial hypertension is a significant risk factor for late cardiovascular morbidity and mortality after repair of coarctation of the aorta. ${ }^{1-4}$ The commonest causes of late death are ruptured aortic aneurysm, cerebrovascular events (atherosclerotic and hypertensive), coronary artery disease and cardiac failure. ${ }^{145}$ While surgical repair usually abolishes the aortic arch gradient, systemic blood pressure often remains abnormal and may increase with time, even in those who became normotensive early after repair. ${ }^{12}$ The mechanisms for these abnormalities of long-term blood pressure regulation remain unclear. Furthermore, treatment in later life may be less effective, as long-term studies have shown persistent hypertension and decreased survival in those who were operated on in adolescence and adulthood. ${ }^{2}$ Residual or recoarctation contributes to hypertension, but abnormalities of the aortic wall, peripheral conduit arteries and resistance vessels, present even after successful repair, ${ }^{6-13}$ may also be important. We have previously demonstrated depressed endothelial and smooth muscle function in the precoarctation vascular bed, in contrast to normal function in the femoral and posterior tibial arteries late after successful repair. ${ }^{78}$ These diffuse abnormalities were associated with higher blood pressure during exercise and normal daily life, ${ }^{67}$ and suggest an acquired element of vascular dysfunction before coarctation repair. However, the relative contribution and importance of residual gradient and the wider pattern of vascular dysfunction as well as the potential for recovery remain unclear.

We therefore designed a study to evaluate the contribution of mechanical aortic obstruction and vascular function to daytime ambulatory systolic blood pressure. We chose adults with coarctation or recoarctation who were likely to have generalised vascular dysfunction. Residual or recoarctation of the aorta can now be relieved successfully by percutaneous transluminal balloon aortoplasty with or without stent insertion. ${ }^{14-16}$ This presented the opportunity to examine the impact of aortic obstruction and wider vascular wall changes on blood pressure control. The potential for normalisation of function in the different vascular beds, and the relationship with blood pressure, are likely to be important predictors of late cardiovascular morbidity and mortality.

\section{PATIENTS AND METHODS}

\section{Study group}

Patients were recruited from the outpatient clinics of the Heart and Great Ormond Street Hospitals, between October 2002 and November 2003. Twelve consecutive adult patients ( $>16$ years) were identified with either a new diagnosis, or past history of coarctation with evidence of hypertension (resting clinic blood pressure $>140$ / $90 \mathrm{~mm} \mathrm{Hg}$ on at least three occasions or 24-hour ambulatory blood pressure $>135 / 85 \mathrm{~mm} \mathrm{Hg}$ ) and/ or a brachial-ankle systolic pressure gradient of $\geqslant 20 \mathrm{~mm} \mathrm{Hg}$ and/or a peak systolic velocity in the aortic arch $>3.5 \mathrm{~m} / \mathrm{s}$ using continuous wave Doppler, with a diastolic tail on transthoracic echocardiography. Suitability for aortic stenting was assessed by cardiac magnetic resonance imaging (stenosis with a peak velocity $>2.5 \mathrm{~m} / \mathrm{s}^{17}$ discrete or single long segment of stenosis, absence of vessel tortuosity, no involvement of major arterial branches-for example, common carotid artery). Written informed consent was obtained from all subjects following approval by the 
Institutional Ethics Committee. To assess normality of baseline vascular function in coarctation subjects, control subjects matched for age and sex were recruited. Twelve healthy, nonsmoking volunteers (hospital medical staff; five male, seven female, age 28 (7) years, mean (SD) blood pressure 110 (14)/64 (7) $\mathrm{mm} \mathrm{Hg}$, receiving no drugs) underwent evaluation of peripheral vascular function, arterial stiffness and central aortic haemodynamics using an identical study protocol. Subsequent analysis was made using serial measurements in coarctation patients after relief of obstruction by aortic stenting.

\section{Study protocol}

Figure 1 summarises the study protocol. All patients underwent vascular function and blood pressure assessment within 14 days before stenting. Daytime systolic blood pressure was assessed using an ambulatory blood pressure (ABP) monitor, and central aortic wall stiffness by pulse wave analysis. ${ }^{18}$ Dilatory capacity of the peripheral conduit arteries measured using right brachial artery flow-mediated, endothelium-dependent dilatation (FMD, a measure of nitric oxide activity) and endothelium-independent dilatation in response to $25 \mu$ g of glyceryl trinitrate (GTN, measurement of smooth muscle function) were recorded. ${ }^{8}$ Forearm vascular resistance was derived from a mathematical equation using mean arterial blood pressure and brachial artery blood flow. All measurements were repeated 2 weeks and 6 months after stenting. Any vasoactive drugs were stopped $>48$ hours before study, and restarted after completion of $A B P$ monitoring. Caffeine was avoided on the day of study, and both patients who smoked were asked to withhold nicotine intake on the day of the study.

\section{Aortic stenting}

This was performed under general anaesthesia. Intra-aortic peak systolic pressure gradients were measured before and after stent deployment. Genesis PG2910P ( $\mathrm{n}=10$, stent length $29 \mathrm{~mm}$ ) and Covered CP 8Zig34 ( $\mathrm{n}=2$, used in native coarctation because of concerns about dissection, stent length $34 \mathrm{~mm}$ ) were used and the procedure was considered successful if the residual intraaortic peak systolic gradient across the coarctation was $\leqslant 20 \mathrm{~mm} \mathrm{Hg} \cdot{ }^{11}$

\section{Blood pressure assessment}

To determine daytime ambulatory systolic blood pressure an oscillometric device (SpaceLabs 5200) was attached to the right arm after completion of the vascular studies. Blood pressure readings were taken every 15 minutes during the day (6:00-23:00).

\section{Vascular function studies}

Studies were performed in each subject in a supine position in a quiet, air-conditioned room $\left(22-25^{\circ} \mathrm{C}\right)$. After resting for $10 \mathrm{~min}$ utes, supine blood pressure in the right arm and leg was measured using an automated oscillometric device (Datascope, Accutor 3). The average of three readings was recorded.

Central aortic function assessment using pulse wave analysis

The augmentation index represents a surrogate marker for aortic stiffness. Arterial wall stiffness was measured using pulse wave analysis performed with applanation tonometry (SphygmoCor pulse wave analysis system; PWV Medical Ltd, Sydney, Australia). The pulse pressure waveform of the radial artery was recorded for 10 seconds and a radial-to-aortic transfer function used to derive the central aortic pulse pressure waveform from which the augmentation gradient and index were determined. ${ }^{18}$ The central aortic pulse pressure waveform peaks twice. The first is caused by left ventricular ejection, and the second is the result of pressure wave reflection from the periphery. The augmentation gradient is the difference between the second and the first peaks, and the augmentation index is the ratio, augmentation gradient:pulse pressure, expressed as a percentage.

\section{Brachial artery vasomotor function}

We used high-resolution ultrasound (Acuson XP10) following a protocol previously described by our group. ${ }^{8}$ A $5-10 \mathrm{MHz}$ linear-array transducer was used to obtain an image of the right brachial artery in longitudinal section $5-10 \mathrm{~cm}$ above the elbow. Assessment of FMD was achieved by measuring the absolute percentage change in brachial artery diameter from baseline in response to reactive hyperaemia induced by inflating a pneumatic cuff to $300 \mathrm{~mm} \mathrm{Hg}$ around the forearm and deflating after 5 minutes. GTN was measured as the change in diameter in response to $25 \mu \mathrm{g}$ of GTN administered sublingually. The vessel baseline blood flow was calculated as the product of the brachial flow velocity time integral, heart rate and the crosssectional area $(\mathrm{ml} / \mathrm{min})$. Forearm vascular resistance was then measured by dividing mean arterial pressure by baseline brachial artery blood flow $(\mathrm{mm} \mathrm{Hg} \cdot \mathrm{min} / \mathrm{ml})$.

\section{Statistical analysis}

Statistical analysis was performed using the SPSS (version 10.1; SPSS Inc, Chicago, Illinois, USA) statistical package. Data are expressed as median (25th, 75th centile) unless otherwise stated. Two sets of analysis were performed on the collected
Figure 1 Study protocol; measurements taken at baseline, 2 weeks and 6 months after stenting.

\section{Study protocol}

Measurements

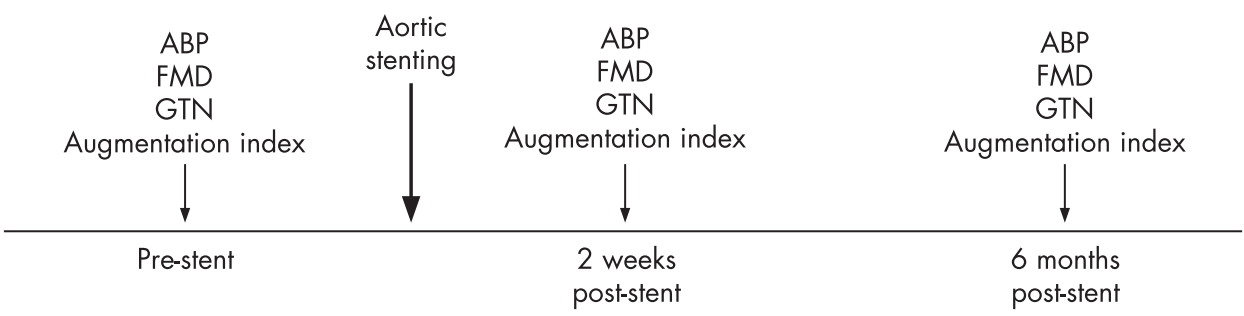


Table 1 Clinical characteristics of the patient group

\begin{tabular}{|c|c|}
\hline Characteristics & Value \\
\hline Male/female & $5 / 7$ \\
\hline Age (years) & $33(19,39)$ \\
\hline Native coarctation & $5(42)$ \\
\hline Recoarctation & $7(58)$ \\
\hline \multicolumn{2}{|l|}{ Type of previous repair:* } \\
\hline End-end anastomosis & $5(42)$ \\
\hline Gortex patch aortoplasty & $1(8)$ \\
\hline Percutaneous transluminal aortoplasty & $1(8)$ \\
\hline \multicolumn{2}{|l|}{ Other cardiac defects: } \\
\hline Bicuspid aortic valve, no functional abnormality & $2(17)$ \\
\hline \multicolumn{2}{|l|}{ Dilated ascending aorta $\dagger$} \\
\hline Severe & $2(17)$ \\
\hline Mild & $1(8)$ \\
\hline \multicolumn{2}{|l|}{ Aortic regurgitation: } \\
\hline Moderate & $1(8)$ \\
\hline Mild & $2(17)$ \\
\hline VSD, spontaneous closure & $1(8)$ \\
\hline Small muscular VSD, no functional abnormality & $1(8)$ \\
\hline VSD, patch repair, and PDA, ligated (infancy) & $1(8)$ \\
\hline \multicolumn{2}{|l|}{ Other comorbidities: } \\
\hline Hepatitis B and C seropositive, no active hepatitis & $1(8)$ \\
\hline Cigarette smoking (1 packet/day) & $2(17)$ \\
\hline Stopped immediately after stent implantation & $1(8)$ \\
\hline
\end{tabular}

Data are expressed as either median (25th, 75th centiles) or number of patients (\% of total).

*Median age of repair 5 years, range 2 days-24 years; median follow-up 19 years, range $17.5-36$ years.

†Ascending aortic dilatation (mild $35-40 \mathrm{~mm}$, severe $>60 \mathrm{~mm}$ ).

\#Aortic regurgitation defined by pressure half time (mild $>500$ ms, moderate 200 $500 \mathrm{~ms}$, severe $<200 \mathrm{~ms}$ ).

PDA, patent ductus arteriosus; VSD, ventricular septal defect.

data. To avoid assumptions about the distribution of the data, we performed two sets of non-parametric analyses to account for any variance. First, coarctation patients and control subjects were compared only at baseline, using the Mann-Whitney U test. A second set of analyses was then performed, comparing within the coarctation group only; between baseline and 2-week follow-up, and between baseline and 6-month follow-up. Data for each variable were compared initially using Friedman two-way analysis of variance testing. Data with significant Friedman test results were further analysed with Wilcoxon matched paired tests. p Values of $<0.05$ were considered significant. Relationships between paired variables were measured using the Spearman rank correlation test. Only the Wilcoxon matched paired test results, rather than Friedman test results, are presented.

\section{RESULTS}

\section{Subjects}

Table 1 shows the clinical characteristics of the patient group. More than half the subjects had previously undergone intervention for coarctation of the aorta, mostly surgery with end-to-end anastomosis (three in infancy). Nearly all (11/12) patients were taking an antihypertensive drug, with over half (58\%) taking more than one agent ( $25 \%$ taking two agents, $8 \%$ three agents, $17 \%$ four agents). Eight patients (67\%) were taking $\beta$-blockers, three $(25 \%)$ thiazide diuretics, four (33\%) ACE inhibitors, one (8\%) indapamide and one (8\%) $\alpha$-methyldopa. Two subjects had bicuspid aortic valves without evidence of left ventricular outflow obstruction, aortic regurgitation or aortic dilatation. Lipid profiles were normal both in patients (cholesterol $4.03(2.20,4.90) \mathrm{mmol} / \mathrm{l}$, triglycerides $0.74(0.51,1.01) \mathrm{mmol} / \mathrm{l})$ and healthy volunteers (cholesterol 4.06 $(2.01,5.00) \mathrm{mmol} / \mathrm{l}$, triglycerides $0.91(0.50,1.20) \mathrm{mmol} / \mathrm{l})$. Subjects did not take statin medication before or after stenting. Heart rate was normal (68 bpm), and did not change at 2 weeks (74 bpm, $p=0.08$ ) and 6 months (71 bpm, $p=0.79)$. One of the two smokers gave up smoking after aortic stenting.

\section{Comparison of vascular measures between subjects with coarctation and controls}

In coarctation subjects at baseline, in comparison with controls, the augmentation index $(26(15,34)$ vs $9(5,14), \mathrm{p}=0.001)$ and forearm vascular resistance $(2.8(2.0,3.2)$ vs $1.2(0.9,1.5)$, $\mathrm{p}=0.02)$ were increased, and there was significant impairment in $\operatorname{FMD}(5.6(2.3,7.1)$ vs $9.0(7.6,10.4), \mathrm{p}=0.001)$ and GTN $(8.7$ $(6.6,9.6)$ vs $12.0(8.2,17.4), \mathrm{p}<0.005)$. Vessel diameter and flow were comparable to those of controls $(p>0.46)$.
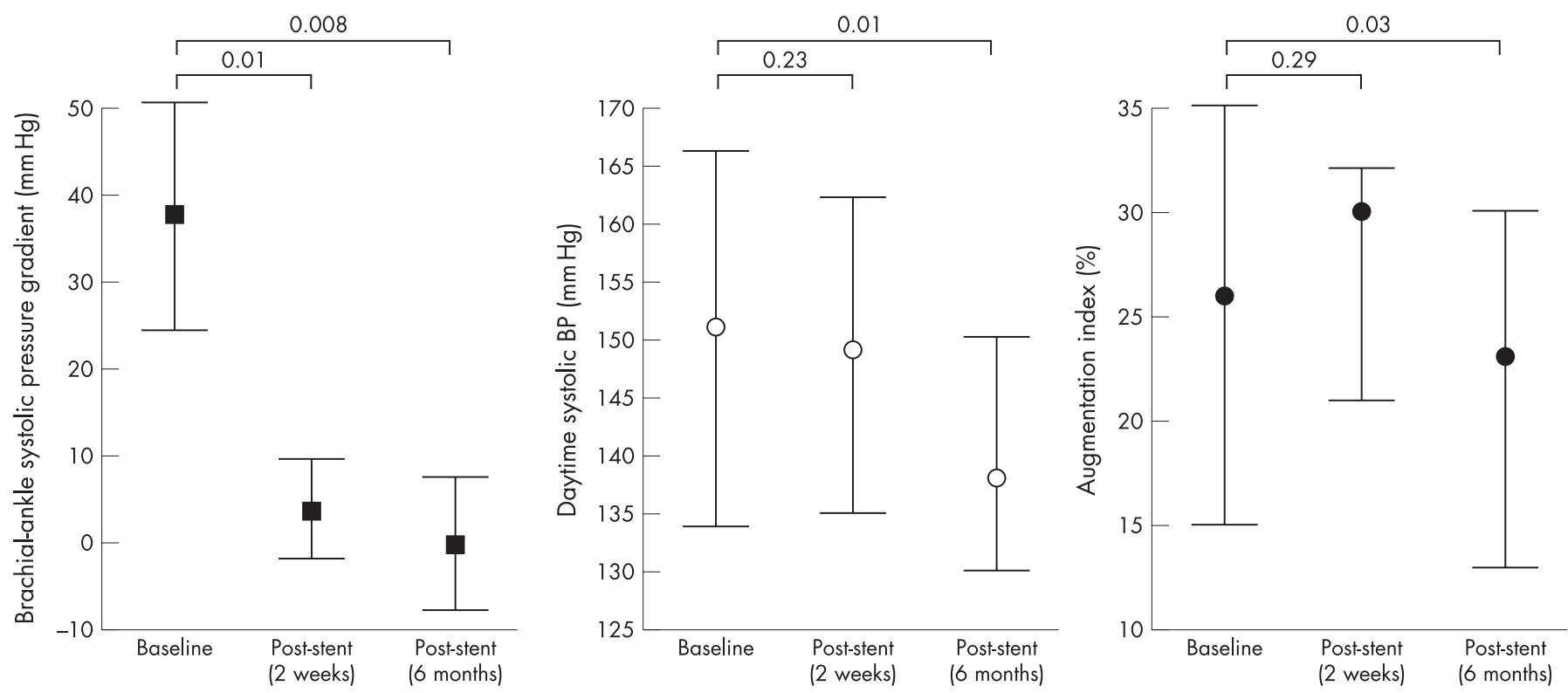

Figure 2 Effect of stenting on ambulatory blood pressure (BP) and central aortic haemodynamics. 
Table 2 Pre- and post-stenting peripheral conduit vascular function

\begin{tabular}{llllll}
\hline Peripheral vascular function & Baseline & $\begin{array}{l}\text { 2 Weeks after } \\
\text { stenting }\end{array}$ & $\begin{array}{l}\text { p Value } \\
\text { (baseline } \\
\text { vs 2 weeks) }\end{array}$ & $\begin{array}{l}\text { 6 Months after } \\
\text { stenting }\end{array}$ & $\begin{array}{l}\text { p Value (baseline } \\
\text { vs } \mathbf{6} \text { months) }\end{array}$ \\
\hline FMD (\%) & $5.6(2.3,7.1)$ & $4.2(2.4,6.6)$ & 0.42 & $3.5(1.6,6.1)$ & 0.79 \\
GTN (\%) & $8.7(6.6,9.6)$ & $8.1(4.7,10.8)$ & 0.72 & $7.7(5.6,8.6)$ & 0.26 \\
$\begin{array}{l}\text { Forearm vascular resistance } \\
\text { (mm Hg.min/ml) }\end{array}$ & $2.8(2.0,3.2)$ & $2.5(2.0,3.4)$ & 1.00 & $2.9(1.5,4.6)$ & 0.89 \\
\hline
\end{tabular}

Data expressed as median (25th, 75th centiles).

$\mathrm{p}$ Values are derived from the Wilcoxon matched paired tests. ${ }^{*} \mathrm{p}<0.05$.

FMD, flow-mediated dilatation; GTN, glyceryl trinitrate.

\section{Effect of stenting on aortic obstruction}

The gradient across the site of coarctation improved immediately after stenting. However, the daytime systolic blood pressure did not fall immediately, and improved after 6 months. This may be due to the augmentation index, which remained abnormal early after stenting, and fell late, at 6 months.

The procedure was effective in relieving obstruction (intraaortic peak systolic gradient under general anaesthesia, before stenting $25(17,46) \mathrm{mm} \mathrm{Hg}$, vs after stenting $6(3,14) \mathrm{mm} \mathrm{Hg}$, $\mathrm{p}=0.002)$. There were no procedural complications.

There was significant improvement in the brachial-ankle systolic pressure gradient 2 weeks after stenting $(37(24,50)$ vs $3(-2,9) \mathrm{mm} \mathrm{Hg}, \mathrm{p}=0.01)$, with a further reduction at 6 months $(-1(-8,7) \mathrm{mm} \mathrm{Hg}, \mathrm{p}=0.008)$.

\section{Effect of stenting on blood pressure and vascular function in coarctation \\ Blood pressure}

Before stenting, all patients were hypertensive during ambulatory monitoring (fig 2). Daytime systolic blood pressure did not change immediately after aortic stenting. However, there was a progressive fall over 6 months (baseline $151(134,166)$ vs 6 months post-stent $138(130,150), p=0.01)$. There were no changes made to the anti-hypertensive drug regimen followed by the subjects during the entire period of study.

\section{Vascular Function \\ Pulse wave analysis}

The augmentation index fell progressively (fig 2), with significant reduction by 6 months (baseline $26(15,34)$ vs 6 months' follow-up $23(13,30), p=0.03)$.

\section{Vasomotor function}

FMD, GTN and forearm vascular resistance remained abnormal 2 weeks and 6 months after stenting (table 2). Baseline vessel diameter and flow also remained unchanged at 2 weeks and 6 months.

\section{Relationships between blood pressure and vascular function}

Daytime systolic blood pressure correlated well with the severity of the intra-aortic pressure gradient $\left(r_{\mathrm{s}}=0.7\right)$, and the augmentation index $\left(r_{\mathrm{s}}=0.5\right)$. There was also an inverse relationship between the daytime systolic blood pressure and GTN $\left(r_{\mathrm{s}}=-0.40\right)$. There were no significant associations with FMD or forearm vascular resistance.

\section{DISCUSSION}

As far as we know, this study is the first to evaluate, prospectively, the impact of stenting aortic coarctation on blood pressure and vascular function. We demonstrate a progressive fall in blood pressure over 6 months after relief of aortic obstruction. This benefit is likely to be due to both acute gradient relief and progressive improvement in central aortic characteristics. Progressive improvement in central aortic characteristics probably contributes to this as blood pressure fell progressively over 6 months after successful relief of aortic obstruction. Residual abnormalities of central and peripheral conduit arterial function, as well as increased vascular resistance in the small vessels, may account for persistent hypertension. Our findings have implications for both monitoring and treatment, as blood pressure is the key determinant of late morbidity and mortality after repair in aortic coarctation. ${ }^{1}{ }^{19}$

There is now more than 50 years of follow-up experience after repair of coarctation and despite the apparent simplicity of the repair, late results have been of concern, ${ }^{12}{ }^{419}$ with increased morbidity and mortality especially from cerebrovascular events, aortic rupture, heart failure and coronary artery disease. ${ }^{45}$ Longterm abnormalities of blood pressure regulation contribute importantly to these complications. ${ }^{1-4}$ We and others have previously shown that systolic blood pressure is increased during exercise and even during normal daily activities. ${ }^{6-9}$ Residual gradient contributes but does not fully account for these findings, and indeed, resting, ambulatory and exerciseinduced systolic hypertension occurs even in the absence of a gradient. ${ }^{6021}$ Blood pressure and vascular changes may be related to age at surgical repair, and it is likely that cumulative vascular abnormalities before intervention contribute to late hypertension. ${ }^{12} 819$ This has been confirmed by histological studies, which have shown decreased smooth muscle and increased collagen mass in the human aorta proximal to the site of coarctation, ${ }^{22}$ as well as persistent medial and intimal hypertrophy in the aortic arch in dogs with coarctation ${ }^{23}$ late after repair. As a result, it has been suggested that late repair, especially in the adult, might not benefit blood pressure. ${ }^{13}$

The availability of percutaneous transluminal balloon aortoplasty and stenting provides an opportunity to investigate the potential improvement in systolic blood pressure after aortic obstruction, as well as the reversibility of generalised vascular function and its relation to blood pressure. The pattern of fall in blood pressure and vascular changes was instructive. Blood pressure did not fall dramatically early after abolition of aortic obstruction, implying that the vascular changes in the aorta, proximal conduit arteries and microvasculature are important determinants. The augmentation index is a composite measure of increased aortic wall stiffness and abnormal wave transmission (both forward and reflected waves), which typically lead to increased systolic and pulse pressures. ${ }^{24}$ In the normal aorta, pressure waves are reflected distally, from the lower part of the body. In coarctation of the aorta, pressure waves are reflected not only from the distal point but also proximally from the site of obstruction, resulting in an increase in the augmentation index. Daytime systolic blood pressure fell as the augmentation index reduced (although not to the level comparable to that of control subjects) over 6 months. Improvement was progressive rather 
than immediate, following gradient relief, implying that the central aortic wall remains stiff, with rapid pressure wave transmission and increased augmentation. The finding of a persistently abnormal aortic physiology is supported by previous experimental and clinical studies, which have shown, using transoesophageal echocardiography, after both surgical repair and balloon dilatation, that the aorta proximal to the site of coarctation remained significantly stiffer than the distal aorta. ${ }^{9-11}$ Furthermore, structural arterial abnormalities are found later in life in those undergoing early repair, even with adequate blood pressure control. ${ }^{12}{ }^{13}$ Studies in a pig model also suggest that abnormal aortic stiffness is inherent to the wall, and is not caused by the stent itself. ${ }^{25}$ Late improvement in central aortic stiffness may represent remodelling of the aortic wall.

We found that both conduit arterial function and small vessel resistance were abnormal and did not improve significantly over the 6-month follow-up. Increased peripheral vascular resistance is a key determinant of blood pressure regulation ${ }^{26}$ and functional conduit artery abnormalities are common ${ }^{27} 28$ in essential hypertension. FMD in the brachial artery may reflect impaired nitric oxide-dependent endothelial dilatation. This is supported by evidence of increased nitric oxide inactivation in a coarctation mouse model. ${ }^{29}$ Endothelial dysfunction is found in other hypertensive populations. It may be both a cause and consequence of hypertension and contribute to atherogenesis and increased risk. ${ }^{30}$ Reduced dilatory ability of the conduit arteries, however, was not solely due to endothelial dysfunction, as the smooth muscle response to GTN also remained depressed. The observation that GTN was inversely correlated with systolic blood pressure supports the concept that impaired smooth muscle dilatation contributes to residual hypertension.

We have performed detailed prospective studies of blood pressure and vascular pathophysiology of a small group of patients from one institution over a 6-month period and evaluated their response to relief of aortic obstruction with a stent. We did not set out to evaluate the clinical, anatomical or surgical determinants of hypertension or vascular dysfunction, which would be an important separate study. Our 6-month study period may have been too short to demonstrate the full potential for normalisation of blood pressure and vascular responses and longer-term evaluation is indicated. Nevertheless, previous work from our group and others has shown that vascular abnormalities can still be detected many years after coarctation relief. ${ }^{6} 81213$ Anti-hypertensive therapy may have a negative effect on vascular remodelling because of its influence on the neurohumoral axis, but we were unable to examine the impact of drug treatment in this study. We did not power the study to detect differences between native and recoarctation patients and larger multiinstitutional study would be required to examine this issue.

Three subjects had aortic regurgitation (two mild and one moderately severe) which did not change in severity after stenting. Although a minor effect on the results of the initial comparisons with healthy subjects cannot be excluded, we would not expect this to have influenced the vascular changes we observed after stenting. It was impractical to test the seven female subjects during a similar stage of the menstrual cycle at each follow-up visit. Although menstrual stage can influence endothelial function, the impact on blood pressure is less consistent and therefore unlikely to have had a major influence on our main findings. Two of our patients were occasional social smokers. Although they did not smoke on the day of the study, one patient did give up smoking after the intervention. While this may have had a small beneficial impact on their vascular function little, if any, impact would be expected on blood pressure.
We have shown that relief of coarctation (native or recoarctation) in adults leads to progressive improvement in blood pressure and vascular measures during normal daily life over a 6-month period. Patients with hypertension and coarctation gradient should therefore be considered for stenting if clinically suitable. It will be important to compare the blood pressure benefit of stenting with anti-hypertensive therapy alone. Hypertension does not completely normalise after intervention, and may be due to residual aortic wall abnormalities, and persistent peripheral vascular dysfunction, emphasising the need for continued surveillance of such patients even after "successful" relief of coarctation.

Acknowledgements: JED and JPH are supported by the British Heart Foundation and AED by CORDA through the Silcock Legacy.

Competing interests: None declared.

Ethics approval: Approved by the Institutional Ethics Committee.

\section{REFERENCES}

1. Cohen M, Fuster V, Steele PM, et al. Coarctation of the aorta: long-term follow-up and prediction of outcome after surgical correction. Circulation 1989;80:840-5

2. Clarkson PM, Nicholson MR, Barratt-Boyes BG, et al. Results after repair of coarctation of the aorta beyond infancy: a 10 to 28 years follow-up with particular reference to late systemic hypertension. Am J Cardiol 1983;51:1541-8.

3. Bhat MA, Neelakandhan M, Unnikrishnan RS, et al. Fate of hypertension after repair of coarctation of the aorta in adults. Br J Surg 2001;88:536-8.

4. Toro-Salazar $\mathbf{0 H}$, Steinberger J, Thomas W, et al. Long-term follow-up of patients after coarctation of the aorta repair. Am J Cardiol 2002;89:541-7.

5. Celemajer DS, Greaves K. Survivors of coarctation repair: fixed but not cured. Heart 2002;88:113-4.

6. de Divitiis M, Pilla C, Kattenhorn M, et al. Ambulatory blood pressure, left ventricular mass, and conduit artery function late after successful repair of coarctation of the aorta. J Am Coll Cardiol 2003;41:2259-65.

7. Gardiner HM, Celermajer DS, Sorensen KE, et al. Arterial reactivity is significantly impaired in normotensive young adults after successful repair of aortic coarctation in childhood. Circulation 1994;89:1745-50.

8. de Divitiis M, Pilla C, Kattenhorn M, et al. Vascular dysfunction after repair of coarctation of the aorta. Impact of early surgery. Circulation 2001;104(suppl I):I-165-I-170.

9. Ong CM, Canter CE, Gutierrez FR, et al. Increased stiffness and persistent narrowing of the aorta after successful repair of coarctation of the aorta: relationship to left ventricular mass and blood pressure at rest and with exercise. Am Heart $J$ 1992;123:1594-600.

10. Brili S, Dernellis J, Aggeli C, et al. Aortic elastic properties in patients with repaired coarctation of the aorta. Am J Cardiol 1998;82:1140-3.

11. Xu J, Shiota T, Omoto R, et al. Intravascular ultrasound assessment of regional aortic wall stiffness, distensibility, and compliance in patients with coarctation of the aorta. Am Heart J 1997:134:93-8.

12. Vriend JWJ, Zwinderman $A H$, de Groot $E$, et al. Predictive value of mild, residual descending aortic narrowing for blood pressure and vascular damage in patients after repair of aortic coarctation. Eur Heart J 2004;26:84-90.

13. Vriend JWJ, de Groot $E$, de Waal $T$, et al. Increased carotid and femoral intimamedia thickness in patients after repair of coarctation: influence of early repair. Am Heart J 2006;151:242-7.

14. Fawzy ME, Awad M, Hassan W, et al. Long-term outcome (up to 15 years) of balloon angioplasty of discrete native coarctation of the aorta in adolescents and adults. J Am Coll Cardiol 2004;43:1062-7.

15. Schrader R, Bussmann WD, Jacobi V, et al. Long-term effects of balloon coarctation angioplasty on arterial blood pressure in adolescent and adult patients. Cardiovasc Diagn 1995;36:220-5

16. Harrison DA, McLaughlin PR, Lazzam C, et al. Endovascular stents in the management of coarctation of the aorta in the adolescent and adult: one year follow up. Heart 2001;85:561-6.

17. Therrien J, Thorne SA, Wright A, et al. Repaired coarctation: a "cost-effective" approach to identify complication in adults. J Am Coll Cardiol 2000;35:997-1002.

18. Yasmin Brown MJ. Similarities and differences between augmentation index and pulse wave velocity in the assessment of arterial stiffness. OJM 1999;92:595-600.

19. Koller M, Rothlin M, Senning A. Coarctation of the aorta: review of 362 operated patients. Long-term follow-up and assessment of prognostic variables. Eur Heart $J$ 1987:8:670-9.

20. Hauser $\mathbf{M}$, Kuehn A, Wilson N. Abnormal responses for blood pressure in children and adults with surgically corrected aortic coarctation. Cardiol Young 2000;10:353-7.

21. Carano V, Agentti A, Barone A, et al. Exercise test in detecting anomalous behaviour of blood pressure in patients successfully operated on for coarctation of the aorta. Pediatr Med Chir 1999;21:105-9.

22. Sehested J, Baandrup U, Mikkelsen E. Different reactivity and structure of the prestenotic aorta in human coarctation. Implications for baroreceptor function. Circulation 1982:65:1060-5. 
23. Leskinen M, Reinila A, Tarkka M, et al. Reversibility of hypertensive vascular changes after coarctation repair in dogs. Pediatr Res 1992;31:297-9.

24. Nichols WW, Singh BM. Augmentation index as a measure of peripheral vascular disease state. Curr Opin Cardiol 2002;17:543-51.

25. Pihkala J, Thyagarajan GK, Taylor GP, et al. The effect of implantation of aortic stent on compliance and blood flow. An experimental study in pigs. Cardiol Young 2001:11:173-81.
26. McVeigh GE, Burns DE, Finkelstein SM, et al. Reduced vascular compliance as a marker of essential hypertension. Am J Hypertens 1991;4:245-51.

27. Taddei S, Virdis A, Mattei P, et al. Endothelium-dependent forearm vasodilation is reduced in normotensive subjects with familial history of hypertension. J Cardiovasc Pharmacol 1992;20(Suppl 12):S193-5

28. Schmieder RE, Weihprecht $\mathrm{H}$, Schobel $\mathrm{H}$, et al. Is endothelial function of the radial artery altered in human essential hypertension? Am J Hypertens 1997;10:323-31.

\section{Images in cardiology}

\section{Acute discrete dissection of the ascending aorta}

A 49-year-old man with a history of hypertension presented to the emergency department because of chest pain and syncope. Acute coronary syndrome was suspected; both ECG and laboratory test were negative. Echocardiography demonstrated a dilated ascending aorta $(56 \mathrm{~mm})$, and a mildly regurgitant bicuspidal aortic valve; neither left ventricular wall motion abnormalities nor pericardial effusion were detected. Aortic dissection was suspected and the patient underwent transoesophageal echocardiography. The study could not identify any intimal flap in the whole thoracic aorta, but attentive inspection of the posterior ascending aorta revealed subtle signs of discrete aortic dissection, namely a limited intimal splitting tear (IT), a discrete adjacent intramural haematoma $(\mathrm{IH})$, and a minimal fluid periaortic effusion (PE). The patient underwent emergent surgery; the echocardiographic findings were confirmed after the ascending aorta was opened; valvesparing surgery (valve reimplantion into a tube graft, according to David technique) was performed. The patient was discharged 1 week later in good general condition; transthoracic echocardiography identified mild residual aortic regurgitation.
The classification of aortic dissection proposed by the European Society of Cardiology is based on anatomic presentation and comprises five classes: 1) classic aortic dissection with true and false lumen separated by an intimal flap; 2) intramural haematoma; 3) subtle or discrete aortic dissection; 4) penetrating atherosclerotic ulcers; and 5) iatrogenic or traumatic dissection. Aortic dissection cannot be ruled out by simply excluding the presence of an intimal flap separating the aorta into two lumina (indicative of class 1 dissection), but attentive inspection of the whole aorta should be performed in order to detect signs typical or suggestive of class 2-4 aortic dissection. In patients with class 3 dissection, attentive inspection of the proximal ascending aorta by transoesophageal echocardiography can provide unique diagnostic information (subtle intimal discontinuity, circumscribed intramural haematoma, discrete periaortic effusion) given the proximity of the aorta to the oesophagus and the millimetric spatial resolution of the technique, leading to prompt emergency surgery, short hospital stay, and good outcome.

\section{F Chirillo, L Salvador, F Bacchion}

Ca' Foncello Hospital, Treviso, Italy

Correspondence to: Ca' Foncello Hospital, Treviso, Italy; fchirillo@ulss.tv.it

Patient consent: Informed consent was obtained for publication of the case details described in this report.

Heart 2008;94:924. doi:10.1136/hrt.2007.115840
Figure 1 Transoesophageal echocardiographic image of the proximal ascending aorta demonstrating subtle typical signs of discrete aortic dissection.

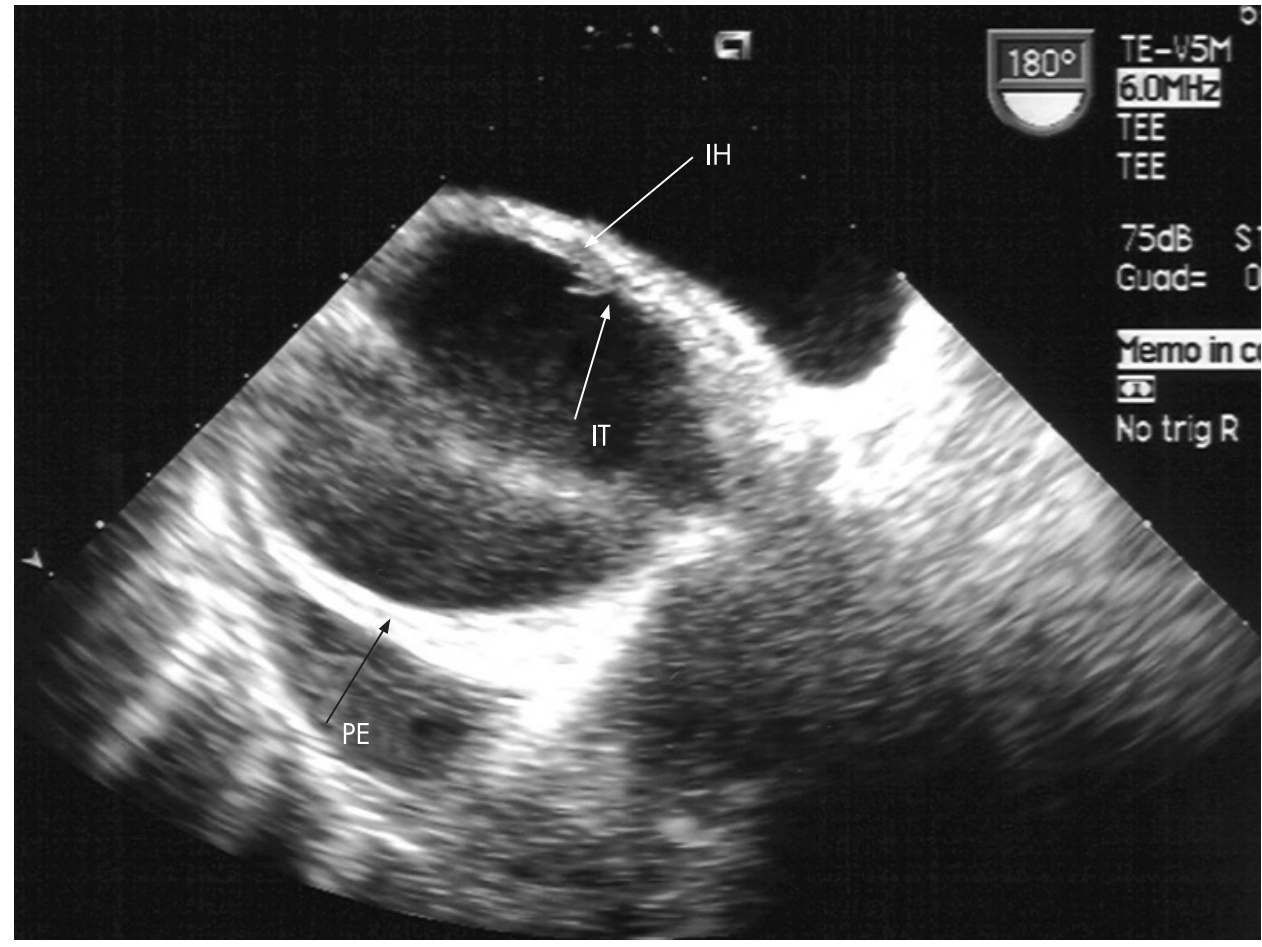

